\title{
$\nabla$ Vaginal Vault Prolapse after Total Hysterectomy in Obese Women
}

\section{IJCRR}

Section: Healthcare

ISI Impact Factor

(2019-20): 1.628

IC Value (2019): 90.81

SJIF (2020) $=7.893$

\section{Dilshod A. Shomirov, Rustem B. Yusupbaev*, Mehriban Dauletova}

Republican Specialized Scientific-Practical Medical Centre of Obstetrics and Gynaecology, Uzbekistan.

(ब) $(\mathbb{8}$

Copyright@IJCRR

\section{ABSTRACT}

Introduction: Total hysterectomy is one of the most common surgical interventions in gynaecology, the frequency of which varies from $30 \%$ to $60 \%$, according to various authors. Moreover, the number of performed extirpation of the uterus has been steadily growing every year. The most common indications for uterine extirpation are uterine fibroids, abnormal uterine bleeding that cannot be treated conservatively, as well as cervical and endometrial cancer.

Objective: Developing effective methods of surgical prevention and correction of vaginal vault prolapse in obese women undergoing a total hysterectomy

Methods: This article describes an individual approach to the prevention of vaginal vault prolapse after total hysterectomy in obese women. The article discusses the features of total hysterectomy in 121 patients with obesity. The author compares 2 surgical tactics for the prevention of apical prolapse after a total hysterectomy.

Results: Long-term results were studied from 2 months to 2 years; the majority of women who operated on the improved technology were satisfied with the results of operations. Everyone noted an improvement in the quality of life related to health.

Conclusion: The proposed parameters of the method allow achieving the set technical result: reducing the risk of early and late postoperative development in hospitals of any level.

Key Words: Total hysterectomy, Obesity, Uterine fibroids, Prolapse

\section{INTRODUCTION}

Genital prolapse is a multi-factorial disease, which necessitates the reasonable choice of a standardized treatment method, on the one hand, and an individual method of surgical correction, on the other hand, for each patient. Vaginal vault prolapse after total hysterectomy is a condition in which the vaginal vault, the bladder, and the anterior wall of the rectum form hernial bulging into the vaginal canal, extending beyond vaginal opening at the later stages of the postoperative period. ${ }^{1,7}$ This condition is not only a medical but also a serious socio-economic and psychological problem that significantly reduces the quality of life of a woman. The significant proportion of vaginal vault prolapse among gynaecological nosology in departments of operative gynaecology, even in highly specialized hospitals, is $19.9-49.6 \%{ }^{2,7,9}$

Only a comprehensive approach will improve the results of surgical treatment of patients with genital prolapse, re- duce the frequency of relapses of this disease and the risk of immediate and long-term adverse results of surgery. ${ }^{6,7}$ The problem is compounded by the fact that almost $2 / 3$ of women with this complication are obese women, who have several technical difficulties during the total hysterectomy due to the pronounced fat layer. ${ }^{2}$ However, the tendency towards obesity among women has been increasing recently. For example, in the United States, the frequency of hysterectomies among laparotomic (abdominal) gynaecological operations is $36 \%$, in Russia from $32.5 \%$ to $38.3 \%$, in Sweden $38 \%$, in the UK-25\%. ${ }^{3,4}$

Vaginal vault prolapse, as a rule, is accompanied by various unpleasant symptoms that cause a woman to experience severe discomfort. Often this process is accompanied by severe pain, urine retention or, conversely, urinary incontinence, frequent urination, problems with defecation. ${ }^{3,8,5} \mathrm{With}$ complete vaginal prolapse after hysterectomy, the mucous

\section{Corresponding Author:}

Rustem B. Yusupbaev, Republican Specialized Scientific-Practical Medical Centre of Obstetrics and Gynaecology, Uzbekistan. Email: rustam.yusupbayev@bk.ru

ISSN: 2231-2196 (Print)

Received: 04.10 .2020
ISSN: 0975-5241 (Online)

Revised: 22.12 .2020
Accepted: 04.02 .2021
Published: 19.05 .2021 
membrane of its walls is subject to severe injuries, which leads to the development of infectious diseases, abscess, and even tissue death. ${ }^{5,9,12}$ Existing methods of surgery do not always radically eliminate the pathology, which makes it necessary to develop new methods of surgery.

In the present study we aimed to develop effective methods of surgical prevention and correction of vaginal vault prolapse in obese women who have undergone total hysterectomy.

\section{MATERIALS AND METHODS}

The study group consisted of 141 women with uterine fibroids more than 12 weeks and obesity that underwent total hysterectomy with abdominal access. At the first stage, there was a comprehensive clinical and laboratory examination of all patients who were admitted for planned surgical treatment for uterine fibroids and with obesity in the Republican specialized scientific-practical medical centre of obstetrics and gynaecology.

Indications for the operation were determined by a diagnosis made based on a comprehensive clinical laboratory examination. In this study the following research methods were used: clinical (interview, examination, gynaecological status), laboratory (examination of general blood and hematocrit before surgery and during the second day after the surgery) and special (examination of the blood clotting sequence). The cultures of the cervical canal, vagina, and urethra were examined.

Patients in the hospital underwent additional examinations, which included ultrasound (trans-vaginal and abdominal access), Doppler study, computed tomography (CT scan), magnetic resonance imaging (MRI), etc.

\section{RESULTS}

Taking into account the method of surgical intervention, all the patients were divided into 2 groups. The main group of 87 women who underwent total hysterectomy operations with laparotomic access using advanced technology attaching the ligamentous apparatus to the vaginal vault, in which the top of the vagina was left open during the operation. The control group consisted of 54 patients who underwent total hysterectomies with laparotomic access using the traditional method. All operations were performed for the purpose of surgical treatment of uterine fibroids more than 12 weeks.

The subjects of both groups were comparable in terms of socio-biological characteristics and severity of the disease.
The study groups included patients with obesity and uterine fibroids more than 12 weeks requiring surgical treatment, aged 45 to 52 years, with an average age of $49.4 \pm 1.78$ years. The decision on the scope of surgical treatment was made individually in each case.

87 operations were performed (the main group) - total hysterectomy with laparotomic access using advanced technology. The essence of the method is to apply a direct clamp simultaneously with the capture of the Sacro-uterine ligaments, cardinal ligaments and vascular bundle at an angle of 45 degrees concerning the conductive axis of the uterus body, which makes it possible to combine the stages of surgery, thereby shortening its duration, reducing the volume of blood loss. Subsequent fixation of Sacro-uterine, cardinal, circular ligaments with the sidewalls of the vagina on each side and simultaneous application of a twisted suture on the front and back walls of vaginal vault is performed with a single thread. Moreover, the beginning of the suture begins with ligation of the vascular bundle on one side, followed by the capture of the cardinal, Sacro-uterine ligaments and the transition to the back wall of the vaginal vault (the beginning of the needle injection into the vaginal vault begins from inside to outside, i.e. from the vaginal mucosa). The opposite side is sewn in reverse order. After ligation of the vascular bundle, it is necessary to fix the circular ligament with the transition to the anterior wall of the vaginal vault (performed by a similar technique). It is necessary to finish the suture by fixing the circular ligament on the opposite side.

Thus, the proposed improved technique of total hysterectomy operation with abdominal access prevents vaginal vault prolapse in obese women. Because when suture is fixed, the size of the Douglass space decreases due to its stretching and lifting, which prevents the further formation of enterocele in obese women. This method preserves the physiological horizontal axis of the vagina in relation to the levators (levatoris ani externa), due to this, sexual function is not disturbed.

The duration of total hysterectomy surgery using advanced technology varied from 45 to 75 minutes, with an average of $52.5+1.5$ minutes.

With the traditional method of abdominal total hysterectomy, the duration of the operation varied from 90 to 110 minutes, $98.6 \pm 5.6$ minutes, $(\mathrm{p}<0.05)$. Thus, in the study group, it averaged $52.5+1.5$ minutes, which was significantly shorter than in the control group - $98.6 \pm 5.6$ minutes, $(p<0.05)$.

As can be seen from Table 1, the size of the uterus according to ultrasound data and the weight of the gross specimen in the compared groups were identical. 
Table 1: Main characteristics in the study groups

\begin{tabular}{lll} 
Characteristics & The study group $(\mathbf{n = 8} 7)$ & The control group $(\mathbf{n = 5 4})$ \\
Uterus size, weeks & $\mathbf{1 7 . 5 \pm 0 . 8}$ & $\mathbf{1 6 . 8 \pm 0 . 5}$ \\
The duration of surgery, min & $52.5 \pm 1.5$ & $98.6 \pm 5.6^{*}$ \\
Weight of the gross specimen, gr & $754 \pm 38.1$ & $768 \pm 26.1$ \\
Total blood loss, ml & $205.6+18.4$ & $290.7 \pm 26.8^{*}$ \\
\hline
\end{tabular}

Note: ${ }^{*} \mathrm{p}<0.05,{ }^{* *} \mathrm{p}<0.01$ significance of differences between groups

The volume of blood loss in the control group was from 250 to $400 \mathrm{ml}$, on average- $290.7 \pm 26.8 \mathrm{ml}$. Significantly less blood loss was registered in the study group - from 200 to $250 \mathrm{ml}$, on average $205.6 \pm 18.4 \mathrm{ml},(\mathrm{p}<0.05)$.

There were no intraoperative complications in the study group. Only in the early postoperative period in $2(1.1 \%)$ cases were subaponeurotic hematomas. After carrying out antibacterial and infusion therapy, the patient recovered.

When performing hysterectomy using the traditional method, that is, in patients of the control group, the following intraoperative complications were registered: $1(0.96 \%)$ injury to the bladder and $1(0.96 \%)$ injury to the ureter. It should be noted that the proposed method of forming the vaginal vault excludes the possibility of accumulation of exudate in the postoperative period, due to the creation of a natural "drainage" of the paravesical and parametric spaces. In this regard, in the study group, leaving the top of the vagina open is not recorded a single case of culcit (Table 2). In contrast, in the early postoperative period, when operating using the traditional method, every tenth patient in the control group - 11 (20.3\%) developed culcit and two cases were diagnosed with subaponeurotic hematomas of the anterior abdominal wall. All cases required infusion, anti-inflammatory and antibacterial therapy in addition to physical therapy.

Table 2: Results of studying the features of the early postoperative course in the examined groups $(\mathrm{M} \pm \mathrm{m})$, people. $(\%)$

\begin{tabular}{|c|c|c|}
\hline $\begin{array}{l}\text { Features of the course of the post- } \\
\text { operative period }\end{array}$ & Study group $(n=87)$ & Control $(n=54)$ \\
\hline Number of patients with a culcit & - & $11(20.3 \%)$ \\
\hline Number of patients with fever & $11(12.6 \%)$ & $42(41.9 \pm 7.8 \%)^{* *}$ \\
\hline \multicolumn{3}{|l|}{ Number of patients with a body temperature figure: } \\
\hline -up to $37.2^{\circ} \mathrm{C}-37 \cdot 4^{\circ} \mathrm{C}$ & $21(24 \%)$ & $14(33 \cdot 3 \%)$ \\
\hline -from $37.5^{\circ} \mathrm{C}$ to $38^{\circ} \mathrm{C}$ & - & $20(48 \%)$ \\
\hline -from $38.2^{\circ} \mathrm{C}$ and above $39{ }^{\circ} \mathrm{C}$ & - & $8(19.04 \%)$ \\
\hline $\begin{array}{l}\text { The number of patients with elevated body tempera- } \\
\text { ture.: } \\
\text {-up to } 3 \text { days }\end{array}$ & $7(8.04 \%)$ & $11(26.2 \%)$ \\
\hline - from 3 to 5 days & - & $28(66.7 \%)$ \\
\hline - from 8 to 10 days & - & $3(7.1 \%)$ \\
\hline Postoperative bed day & $4.7+1.1$ & $8.4 \pm 1.3^{*}$ \\
\hline
\end{tabular}

Note: ${ }^{*} \mathrm{p}<0.05,{ }^{* *} \mathrm{p}<0.01$ significance of differences between groups.

The duration of stay of patients in the hospital was 4-6 days. The average blood loss of $180.4+3,3 \mathrm{ml}$. There were no intraoperative complications. In the early postoperative period, $2(2.3 \%)$ cases had subgaleal hematomas. After carrying out antibacterial and infusion therapy, the patients recovered. The duration of the operation varied from 90 to 110 minutes with the traditional method of abdominal hysterectomy. The duration of patients ' stay in the hospital was 5-7 days. The average blood loss was $290.7 \pm 26.8 \mathrm{ml}$.
In the course of total hysterectomy according to the traditional method, the vagina is shortened, and therefore, sex life becomes difficult. When performing a hysterectomy using laparotomic access using the traditional method, the following complications were registered: intraoperatively-1 (1.9\%) injury to the bladder and $1(1.9 \%)$ injury to the ureter. In the early postoperative period, $2(3.7 \%)$ periculitis, granulomas in every third patient, and $3(5.5 \%)$ subaponeurotic hematomas of the anterior abdominal wall 
were detected. After carrying out antibacterial and infusion therapy, the patients recovered.

\section{DISCUSSION}

As with any surgical intervention, after extirpation of the uterus, early and late postoperative complications are possible. The most common early complication after extirpation of the uterus is a temperature reaction and suppuration. This type of complication is caused by the frequent accumulation of a small amount of exudate in the Douglas space. In the proposed method of forming a vaginal vault, the possibility of accumulation of exudate in the postoperative period is excluded, due to the creation of a natural "drainage" of the paravesical and parametral spaces. Leaving the top of the vaginal open, we did not observe such complications, which contributed to the earliest possible discharge from the hospital. The improved technique of suturing the anterior and posterior walls of the vagina, vault of Sacro-uterine, round and cardinal ligaments, allows minimizing the number of postoperative complications. ${ }^{7,8,9}$

To confirm the effectiveness of the improved method of suturing vaginal vault we have analyzed the various characteristic features of the early postoperative period in patients who underwent surgery of total hysterectomy using improved methods and traditional methods such as number of bed-days after surgery, the number of days with increased body temperature, the figures of the maximum rise of a body temperature and the number of women with culcit and granuloma. ${ }^{10,11}$

The vast majority of patients who underwent surgery using the improved method $92.1 \%$ - were in the hospital after the operation for up to 5 days, while women operated using the traditional method- $66.3 \%$. Also, in patients who underwent surgery using an improved technique, granulomas were not observed, which occurred in every third operated by the traditional method. Thus, the obtained results show the advantage of using the improved method of creating artificial drainage of the postoperative wound: reducing complications in the form of culcit, the formation of granulomas, reducing the number of days with fever, reducing drug use, reducing patients ' stay in hospital and increasing the turnover of beds. ${ }^{5,12}$

Restrictions in the process of sexual life are absent in the group of women operated on by improved technology because, during the operation, the ligaments were attached to the vagina vault, which formed the top of the vagina, the physiological horizontal axis of the vagina about the levators is preserved, which creates the possibility of normal sexual life. In the course of a traditional total hysterectomy, the vagina is shortened, which makes it difficult to have sex.,6
When observed for 2 years after the operation using an improved technique, during which the vaginal vault was fixed with Sacro-uterine, cardiac, and circular ligaments, there was no vaginal vault prolapse. Whereas, in women who underwent surgery using the traditional method, vaginal vault prolapse was observed in 17 patients, including cystocele in 9 cases, rectocele in 3 cases, and enterocele in 5 cases. ${ }^{3,12}$ Long-term results were studied from 2 months to 2 years; the majority of women who operated on the improved technology were satisfied with the results of operations. Everyone noted an improvement in the quality of life related to health. The proposed parameters of the method allow achieving the set technical result: reducing the risk of early and late postoperative development in hospitals of any level.

\section{CONCLUSIONS}

Thus, our proposed improved technique of total hysterectomy operation prevents vagina vault prolapse in obese women. Because when the seam is fixed, the size of the Douglass space decreases due to its stretching and lifting, which prevents the further formation of enterocele in obese women. This method preserves the physiological horizontal axis of the vagina about the levators (levatoris ani externa), due to this, sexual function is not disturbed.

In the proposed method of forming the vagina vault, the possibility of accumulation of exudate in the postoperative period is excluded, due to the creation of a natural "drainage" of the paravesical and parameter spaces.

Conflict of interest: No

Author's contributions: This work was carried out in collaboration among all authors. Shomirov Dilshod conducted operations and wrote the first draft of the manuscript. Yusupbaev Rustem designed the study, performed the statistical analysis, wrote the protocol, and. Dauletova Mehriban managed the analyses of the study and the literature searches. All authors read and approved the final manuscript.

Funding: The research was carried out in Republican specialized scientific-practice medical centre of Obstetrics and Gynecology within the framework of the applied research project: "Development of a surgical method for the prevention and treatment of vaginal prolapses after total hysterectomy in obese women"

\section{REFERENCES}

1. Korshunov MY. Effectiveness of vaginal hysterectomy with the high suspension of the vaginal dome to the Sacro-uterine ligaments in the treatment of pelvic organ prolapse in women: scientific publication. Russian Bull Obstetri Gynec 2015;13(2):61-65. 
2. Navruzov BS. Diagnostics of rectocele in pelvic organ prolapse in women: scientific publication. Bull Ass Phys Uzbekis 2014; $1: 45-47$

3. Gasparov AS, Dubinskaya ED, Babicheva IA, Lapteva NV. Role of connective tissue dysplasia in obstetric and gynaecological practice. Kazan Med J 2014;95(6):897-904.

4. Dovlatov ZA, Dovlatov ZA. Mesh implants in the treatment of pelvic organ prolapse in women: complications and ways to prevent them. Mod Probl Sci Edu 2015;5:351.

5. Plekhanov AN, Strezelecki VV. minimally Invasive approaches in the surgical treatment of patients with uterine fibroids larger sizes. Moscow Surg J 2008;2(2):11-17.

6. Puchkov KV, Ivanov VV, Bakov VS, Usachev IA. Optimization of the technique of surgical treatment of pelvic prolapse. minimally Invasive technologies in surgery: materials Mezhregion. scientific-practical conf. Makhachkala 2005;12:159-160.

7. Fedorov AA. Influence of hysterectomy on the anatomical and functional state of the urinary system. Abstract. Diss. for the Degree of Doctor of Science 2005;5:8-16.
8. Shalaev ON, Radzinsky VE, Plaksina ND, Salimova LY. Fixation of the vagina to the Sacro-spinous ligament as prevention of recurrent genital prolapse. Bulletin of the Peoples' Friendship University of Russia, Moscow. 2017;7:17-18.

9. Ghetti C. Pelvic organ descent and symptoms of pelvic floor disorders. Am J Obstet Gynecol 2018;7:53-57.

10. Gasparov AS, Babicheva IA, Dubinskaya ED, Lapteva NV, Dorfman MF. Surgical treatment of pelvic organ prolapse. Kazan Med J 2014;95(3):341-347.

11. Mgeliashvili MV, Buyanova SN, Marchenko TB, Rizhinashvili ID. Experience in the use of synthetic prostheses for the treatment of severe forms of genital prolapse in elderly women. Almanac of Clin Med 2015;37:118-122.

12. Moroz NV. Ultrasound in the assessment of the pelvic floor. Obstet Gynec 2015;14(2):31-37. 